\title{
THE CORONA PROPERTY FOR BOUNDED ANALYTIC FUNCTIONS IN SOME BESOV SPACES
}

\author{
ARTUR NICOLAU
}

(Communicated by Clifford J. Earle, Jr.)

\begin{abstract}
In this paper, the corona theorem for the algebra of bounded analytic functions in the unit disc which are in the Besov space $B_{p}, 1<p<\infty$, is proved.
\end{abstract}

Let $\Delta$ be the open unit disc in the complex plane and let $H^{\infty}$ be the Banach space of all bounded analytic functions on $\Delta$ with the norm

$$
\|f\|_{\infty}=\sup \{|f(z)|: z \in \Delta\} .
$$

For $1<p<\infty$, let $B_{p} A$ be the class of all analytic functions $f$ on $\Delta$ such that

$$
\|f\|_{B_{p}(\Delta)}^{p}=\frac{1}{\pi} \int_{\Delta}\left|f^{\prime}(z)\right|^{p}(1-|z|)^{p-2} d m(z)<\infty .
$$

It is easy to see that $H^{\infty} \cap B_{p} A$ is a Banach algebra with the norm $\|f\|=$ $\|f\|_{\infty}+\|f\|_{B_{p}(\Delta)}$. In this note we consider the corona problem for this algebra.

Let $\mathscr{M}$ be the maximal ideal space of $H^{\infty} \cap B_{p} A$ endowed with the Gelfand topology. It is clear that $\Delta$ is naturally embedded in $\mathscr{M}$. The corona problem consists of knowing if $\Delta$ is dense in $\mathscr{M}$. Here we answer this question in the affirmative. As is known (see [2, p. 191]), this turns out to be equivalent to the following result.

Theorem. Let $1<p<\infty$. Given $f_{1}, \ldots, f_{n} \in H^{\infty} \cap B_{p} A$ such that

$$
\max _{j}\left|f_{j}(z)\right| \geq \delta>0, \quad z \in \Delta
$$

there exist $g_{1}, \ldots, g_{n} \in H^{\infty} \cap B_{p} A$ such that

$$
f_{1} g_{1}+\cdots+f_{n} g_{n}=1
$$

Received by the editors May 22, 1989.

1980 Mathematics Subject Classification (1985 Revision). Primary 30A98; Secondary 46J15, $46 \mathrm{~J} 20$.

Key words and phrases. Corona theorem, Besov space. Spain.

Supported in part by the grant PB85-0374 of the CICYT, Ministerio de Educacion y Ciencia, 
Note that for $p=2, H^{\infty} \cap B_{2} A$ is the space of bounded analytic functions with finite Dirichlet integral. So our result contains the answer to a question of [3].

Proof of the theorem. By a normal families argument, we can assume that the corona data $f_{1}, \ldots, f_{n}$ are analytic on a neighborhood of the closed unit disk, and we have to find analytic functions $g_{1}, \ldots, g_{n}$ satisfying (2) and

$$
\left\|g_{i}\right\| \leq C \quad i=1, \ldots, n,
$$

where $C$ is a constant depending on $\delta,\left\|f_{1}\right\|, \ldots,\left\|f_{n}\right\|$.

It is clear that

$$
\varphi_{i}(z)=\overline{f_{i}(z)} / \sum_{i=1}^{n}\left|f_{i}(z)\right|^{2}
$$

is a nonanalytic solution of (2).

As in the case of $H^{\infty}$ (see [2, Chapter VIII]), our problem is equivalent to solving, with bounds, the following equations. For $1 \leq j, k \leq n$, find $b_{j, k}$ such that

$$
\bar{\partial} b_{j, k}=\varphi_{j} \bar{\partial} \varphi_{k} \text { in } \Delta
$$

with

$$
\left\|b_{j, k}\right\|_{L^{\infty}(\mathbf{T})}+\int_{\Delta}\left|\nabla b_{j, k}(z)\right|^{p}(1-|z|)^{p-2} d m(z) \leq C,
$$

where $C$ is a constant depending on $\delta,\left\|f_{1}\right\|, \ldots,\left\|f_{n}\right\|$.

It is sufficient to deal with an equation $\bar{\partial} b=g$ where $g=\varphi_{j} \bar{\partial} \varphi_{k}$. Applying (1), a calculation (see $[2$, p. 326]) gives

$$
|g(z)| \leq M \sum_{j=1}^{n}\left|f_{j}^{\prime}(z)\right|
$$

where $M$ is a constant depending on $\delta$.

In order to find a solution of (3) with bounded $L^{\infty}(\mathbf{T})$ norm it suffices to show that $|g(z)| d m(z)$ is a Carleson measure (see [2, p. 320]). Let us see that this is true.

Put $Q_{h}=\{z \in \Delta:|z| \geq 1-h$ and $\theta-h \leq \operatorname{Arg} z \leq \theta+h\}$. From (5) one has 


$$
\begin{aligned}
\int_{Q_{h}}|g(z)| d m(z) \leq & M \sum_{j=1}^{n} \int_{Q_{h}}\left|f_{j}^{\prime}(z)\right| d m \\
= & M \sum_{j=1}^{n} \int_{Q_{h}}\left|f_{j}^{\prime}(z)\right|(1-|z|)^{1-2 / p}(1-|z|)^{-(1-2 / p)} d m(z) \\
\leq & M \sum_{j=1}^{n}\left[\int_{Q_{h}}\left|f_{j}^{\prime}(z)\right|^{p}(1-|z|)^{p-2} d m(z)\right]^{1 / p} \\
& \times\left[\int_{Q_{h}}(1-|z|)^{-(1-2 / p) p /(p-1)} d m(z)\right]^{(p-1) / p} \\
\leq & (p-1) M \sum_{j=1}^{n}\left(\int_{\Delta}\left|f_{j}^{\prime}(z)\right|^{p}(1-|z|)^{p-2} d m(z)\right)^{1 / p} h \\
\leq & (p-1) M n \sup _{i}\left\|f_{i}\right\| \cdot h .
\end{aligned}
$$

And so, (3) can be solved by means of bounded functions and one has

$$
\inf \left\{\|H\|_{L^{\infty}(\mathbf{T})}: H \text { solves }(3)\right\} \leq C .
$$

In order to obtain a solution of (3) bounded with respect to the norm

$$
\|b\|_{B_{p}(\Delta)}^{p}=\int_{\Delta}|\nabla b(z)|^{p}(1-|z|)^{p-2} d m(z)
$$

let us take

$$
H_{0}(z)=\frac{1}{\pi} \int_{\Delta} \frac{g(\xi)}{\xi-z} d m(\xi) .
$$

One has $\bar{\partial} H_{0}=g$ in $\Delta$. So, applying (5),

$$
\int_{\Delta}\left|\bar{\partial} H_{0}(z)\right|^{p}(1-|z|)^{p-2} d m(z) \leq C .
$$

Furthermore, $\partial H_{0}$ is the Beurling transform of $g$. Since $(1-|z|)^{p-2}$ is an $A_{p}$ weight for $1<p<\infty$ (see [1, p. 411]), one has

$$
\begin{aligned}
& \int_{\Delta}\left|\partial H_{0}(z)\right|^{p}(1-|z|)^{p-2} d m(z) \\
& \quad \leq K(p) \int_{\Delta}|g(z)|^{p}(1-|z|)^{p-2} d m(z) \leq K(p) C
\end{aligned}
$$

because of (5). So

$$
\left\|H_{0}\right\|_{B_{p}(\Delta)} \leq C \text {. }
$$

Nevertheless, the problem is to solve the $\bar{\partial}$ equation (3) by means of a function $b$ satisfying simultaneously the two bounds

$$
\|b\|_{L^{\infty}(\mathbf{T})} \leq C \text { and }\|b\|_{B_{p}(\Delta)} \leq C .
$$


To do this, for $1<p<\infty$, let us consider the Besov class $B_{p}(\mathbf{T})$ formed by those functions in $L^{p}(\mathbf{T})$ such that

$$
\|f\|_{B_{p}(\mathbf{T})}^{p}=\int_{-\pi}^{\pi} \frac{1}{h^{2}} \int_{-\pi}^{\pi}\left|f\left(e^{i(t+h)}\right)-f\left(e^{i t}\right)\right|^{p} d t d h<\infty .
$$

If $f \in L^{p}(\mathbf{T})$ and $u$ denotes its Poisson integral, it is well known (see [5, p. 152]) that there exist an absolute constant $M$ such that

$$
\begin{aligned}
M^{-1} \int_{\Delta}|\nabla u(z)|^{p}(1-|z|)^{p-2} d m(z) & \leq\|f\|_{B_{p}(\mathbf{T})} \\
& \leq M \int_{\Delta}|\nabla u(z)|^{p}(1-|z|)^{p-2} d m(z) .
\end{aligned}
$$

Claim. $\left\|H_{0}\right\|_{B_{p}(\mathbf{T})} \leq C$.

Of course, since $H_{0}$ is not harmonic, the claim cannot be deduced automatically from (7) and (8). Assume the claim is true and let us finish the proof of the theorem.

Since $\bar{\partial} H_{0}=g$ and (6), one has

$$
\inf \left\{\left\|H_{0}-F\right\|_{\infty}: F \in \mathrm{BMOA}\right\}=\inf \left\{\|H\|_{\infty}: H \text { solves }(3)\right\} \leq C,
$$

where BMOA is the space of analytic functions on $\Delta$ with boundary values of bounded mean oscillation.

Peller and Hruscev proved that $B_{p}(\mathbf{T})$ has the best approximation property, for $1<p<\infty$ (see [4, p. 103]). So, there exists a unique $F_{0} \in$ BMOA satisfying

$$
\left\|H_{0}-F_{0}\right\|_{\infty}=\inf \left\{\left\|H_{0}-F\right\|_{\infty}: F \in \mathrm{BMOA}\right\} \leq C
$$

and furthermore

$$
\left\|F_{0}\right\|_{B_{p}(\mathbf{T})} \leq K\left\|H_{0}\right\|_{B_{p}(\mathbf{T})} .
$$

Therefore $H_{0}-F_{0}$ is a solution of the $\bar{\partial}$ equation (3), satisfying $\left\|H_{0}-F_{0}\right\|_{\infty} \leq$ $C$. Now, apply (7), (8), (9), and the claim to get

$$
\begin{aligned}
& \left(\int_{\Delta}\left|\nabla\left(H_{0}-F_{0}\right)(z)\right|^{p}(1-|z|)^{p-2} d m(z)\right)^{1 / p} \\
& \leq\left(\int_{\Delta}\left|\nabla H_{0}(z)\right|^{p}(1-|z|)^{p-2} d m(z)\right)^{1 / p} \\
& \quad+2\left(\int_{\Delta}\left|F_{0}^{\prime}(z)\right|^{p}(1-|z|)^{p-2} d m(z)\right)^{1 / p} \\
& \leq C .
\end{aligned}
$$

So $H_{0}-F_{0}$ satisfies (3) and (4).

Proof of the claim. First of all, we remark that

$$
\left\|H_{0}\right\|_{\mathrm{BMO}(\mathbf{T})} \leq C
$$


with the constant $C$ depending only on the data of the corona problem. Because of [6, Theorem 1.1.2.], one only has to check that $\left|\nabla H_{0}(z)\right| d m(z)$ is a Carleson measure with norm only depending on $\delta,\left\|f_{1}\right\|, \ldots,\left\|f_{n}\right\|$, and in fact, this has been done in the proof of (6).

To prove the claim, we have to show that

$$
\int_{-\pi}^{\pi} \frac{1}{h^{2}} \int_{-\pi}^{\pi}\left|H_{0}\left(e^{i(s+h)}\right)-H_{0}\left(e^{i s}\right)\right|^{p} d s d h \leq C .
$$

Since $\left\|H_{0}\right\|_{\mathrm{BMO}(\mathbf{T})} \leq C$, one has $\int_{-\pi}^{\pi}\left|H_{0}\left(e^{i \theta}\right)\right|^{p} d \theta \leq A C$ where $A$ is an absolute constant. Therefore, by symmetry on $h$, in order to prove (10), it suffices to verify

$$
\int_{0}^{1 / 2} \frac{1}{h^{2}} \int_{-\pi}^{\pi}\left|H_{0}\left(e^{i(s+h)}\right)-H_{0}\left(e^{i s}\right)\right|^{p} d s d h \leq C .
$$

Let us just reproduce a proof of the second inequality in (8) and let us see that the harmonicity is not used.

Take $r=1-h$ and let $\partial H_{0} / \partial n, \partial H_{0} / \partial \theta$ be the derivatives of $H_{0}$ with respect to the radius and the argument. We have

$$
\begin{aligned}
& \mid H_{0}\left(e^{i(s+h)}-H_{0}\left(e^{i s}\right) \mid\right. \\
& \leq\left|H_{0}\left(e^{i(s+h)}\right)-H_{0}\left(r e^{i(s+h)}\right)\right|+\left|H_{0}\left(r e^{i(s+h)}\right)-H_{0}\left(r e^{i s}\right)\right| \\
& \quad+\left|H_{0}\left(r e^{i s}\right)-H_{0}\left(e^{i s}\right)\right| \\
& \leq \int_{r}^{1}\left|\frac{\partial H_{0}}{\partial n}\left(\xi e^{i(s+h)}\right)\right| d \xi+\int_{0}^{h}\left|\frac{\partial H_{0}}{\partial \theta}\left(r e^{i(s+\varphi)}\right)\right| d \varphi+\int_{r}^{1}\left|\frac{\partial H_{0}}{\partial n}\left(\xi e^{i s}\right)\right| d \xi .
\end{aligned}
$$

Apply Minkowski integral inequality [5, p. 271], to get

$$
\begin{aligned}
\left(\int_{-\pi}^{\pi} \mid\right. & \left.H_{0}\left(e^{i(s+h)}\right)-\left.H_{0}\left(e^{i s}\right)\right|^{p} d s\right)^{1 / p} \\
\leq & \int_{r}^{1}\left(\int_{-\pi}^{\pi}\left|\frac{\partial H_{0}}{\partial n}\left(\xi e^{i(s+h)}\right)\right|^{p} d s\right)^{1 / p} d \xi \\
& +\int_{0}^{h}\left(\int_{-\pi}^{\pi}\left|\frac{\partial H_{0}}{\partial \theta}\left(r e^{i(s+\varphi)}\right)\right| d s\right)^{1 / p} d \varphi \\
& +\int_{r}^{1}\left(\int_{-\pi}^{\pi}\left|\frac{\partial H_{0}}{\partial n}\left(\xi e^{i s}\right)\right|^{p} d s\right)^{1 / p} d \xi \\
= & 2 \int_{r}^{1}\left(\int_{-\pi}^{\pi}\left|\frac{\partial H_{0}}{\partial n}\left(\xi e^{i s}\right)\right|^{p} d s\right)^{1 / p} d \xi+h\left(\int_{-\pi}^{\pi}\left|\frac{\partial H_{0}}{\partial \theta}\left(r e^{i s}\right)\right|^{p} d s\right)^{1 / p} \\
= & (I)+(I I) .
\end{aligned}
$$


Changing to planar coordinates and applying (7), one gets

$$
\begin{aligned}
\int_{0}^{1 / 2} \frac{1}{h^{2}}(I I)^{p} d h & =\int_{0}^{1 / 2} h^{p-2} \int_{-\pi}^{\pi}\left|\frac{\partial H_{0}}{\partial \theta}\left(r e^{i s}\right)\right|^{p} d s d h \\
& =\int_{0}^{1 / 2} h^{p-2} \int_{-\pi}^{\pi}\left|\frac{\partial H_{0}}{\partial \theta}\left((1-h) e^{i s}\right)\right|^{p} d s d h \\
& \leq 2 \int_{\Delta}\left|\nabla H_{0}(z)\right|^{p}(1-|z|)^{p-2} d m(z) \leq C .
\end{aligned}
$$

For the term $(I)$, put $x=1-\xi$ and apply Hardy's inequality ([5, p. 272]) to obtain

$$
\begin{aligned}
\int_{0}^{1 / 2} \frac{1}{h^{2}}(I)^{p} d h & =2^{p} \int_{0}^{1 / 2} \frac{1}{h^{2}}\left[\int_{r}^{1}\left(\int_{-\pi}^{\pi}\left|\frac{\partial H_{0}}{\partial n}\left(\xi e^{i s}\right)\right|^{p} d s\right)^{1 / p} d \xi\right]^{p} d h \\
& =2^{p} \int_{0}^{1 / 2} \frac{1}{h^{2}}\left[\int_{0}^{h}\left(\int_{-\pi}^{\pi}\left|\frac{\partial H_{0}}{\partial n}\left((1-x) e^{i s}\right)\right|^{p} d s\right)^{1 / p} d x\right]^{p} d h \\
& \leq 2^{p} K(p) \int_{0}^{1 / 2} h^{-2+p} \int_{-\pi}^{\pi}\left|\frac{\partial H_{0}}{\partial n}\left((1-h) e^{i s}\right)\right|^{p} d s d h \\
& \leq 2^{p+1} K(p) \int_{\Delta}\left|\nabla H_{0}(z)\right|^{p}(1-|z|)^{p-2} d m(z) \leq C
\end{aligned}
$$

because of (7).

This gives (10) and therefore we have proved the claim. This completes the proof of the theorem.

\section{ACKNOWLEDGMENT}

I am grateful to Julià Cufi for many helpful conversations.

\section{REFERENCES}

1. J. Garcia-Cuerva and J. L. Rubio de Francia, Weighted norm inequalities and related topics, North-Holland, Amsterdam, 1985.

2. John B. Garnett, Bounded analytic functions, Academic Press, New York, 1981.

3. Kwang-Nan Chow and David Protas, The maximal ideal space of bounded, analytic, Dirichlet finite functions, Arch. Math. (Basel) 31 (1978/79), 301.

4. V. V. Peller and S. V. Hruscev, Hankel operators, best approximations, and stationary Gaussian processes, Russian Math. Surveys 37 (1982), 103.

5. Elias M. Stein, Singular integrals and differentiability properties of functions, Princeton University Press, Princeton, NJ, 1970.

6. N. Th. Varopoulos, BMO functions and the $\bar{\partial}$-equation, Pacific J. Math. 71 (1977), 223.

Departament de Matemàtiques, Universitat Autònoma de Barcelona, 08193 BellaTERRA, Barcelona, SPain 